\title{
Extranodal Extension in Lymph Node-Positive Prostate Cancer
}

Liang Cheng, M.D., Thomas M. Pisansky, M.D., Dharamdas M. Ramnani, M.D., Bradley C. Leibovich, M.D., John C. Cheville, M.D., Jeff Slezak, B.S., Erik J. Bergstralh, M.S., Horst Zincke, M.D., Ph.D, David G. Bostwick, M.D.

Departments of Pathology and Urology, Indiana University School of Medicine (LC), Indianapolis, Indiana; the Division of Radiation Oncology (TMP), the Department of Pathology (JCC), the Department of Urology (BCL, HZ), and the Section of Biostatistics (JS, EJB), Mayo Clinic and Mayo Foundation, Rochester, Minnesota; and Bostwick Laboratories (DMR, DGB), Richmond, and University of Virginia Health Sciences Center (DGB), Charlottesville, Virginia

Evaluation of extranodal tumor extension may provide prognostic information for patients with epithelial malignancies. However, its importance for the patient who has prostate cancer with regional lymph node metastasis requires further investigation and clarification. This study was performed to evaluate the prognostic significance of extranodal extension (ENE) in a large series of node-positive patients. The study group included 212 nodepositive patients who were treated by bilateral pelvic lymphadenectomy, radical retropubic prostatectomy, and androgen deprivation between 1987 and 1992 at the Mayo Clinic. ENE was defined as cancer perforating through the lymph node capsule into perinodal tissue. Nodal cancer volume was measured by the grid method. Univariate and multivariate risk ratios (RR) for distant metastasis-free and cancer-specific survival were estimated using the Cox proportional model. The mean follow-up was 6.3 years (median, 6.1 years). Distant metastasisfree and cancer-specific survival at 5 years for all patients was $91 \%$ and $95 \%$, respectively. ENE was found in 126 of 212 patients (59\%). The presence of ENE was not significantly associated with distant metastasis-free $(R R=1.6 ; 95 \%$ confidence interval $[C I], 0.7$ to 3.9) or cancer-specific survival $(R R=2.2$; 95\% CI, 0.7 to 6.8). Among 98 patients with a single positive node, there was no significant difference in distant metastasis or cancer-specific survival according to the presence of ENE $(P=.88$ and $P=.36$, respectively). After adjusting for Gleason score, DNA

Copyright () 2000 by The United States and Canadian Academy of Pathology, Inc.

VOL. 13, NO. 2, P. 113, 2000 Printed in the U.S.A.

Date of acceptance: August 25, 1999.

Address reprint requests to: Liang Cheng, M.D., Department of Pathology, UH 3465, Indiana University School of Medicine, 550 North University Boulevard, Indianapolis, IN 46202; e-mail: lcheng@iupui.edu; fax: 317274-5346. ploidy, and ENE, only nodal cancer volume was significantly associated with adverse distant metastasis-free $(\mathrm{RR}=1.9 ; 95 \% \mathrm{CI}, 1.5$ to 2.8$)$ and cancer-specific survival $(\mathrm{RR}=1.4 ; 95 \% \mathrm{CI}, 1.1$ to 1.9). Our data indicate that the presence of ENE is not associated with unfavorable survival in patients with node-positive prostate cancer treated by radical retropubic prostatectomy, bilateral pelvic lymphadenectomy, and androgen deprivation therapy. In contrast, nodal cancer volume was predictive of distant metastasis-free survival and cancerspecific survival.

KEY WORDS: Extranodal extension, Metastasis, Neoplasm, Prognosis, Prostate, Staging, Survival. Mod Pathol 2000;13(2):113-118

The treatment of patients who have prostate cancer with regional lymph node metastasis is controversial (1-7). Although treatment may not cure such patients, regional lymph node metastasis may be the only manifestation of cancer progression in some patients $(8,9)$. Identification of those who are at greatest risk for developing distant metastasis and mortality from prostate cancer will help stratify patients into prognostically distinct groups. Previous studies demonstrated that assessment of extranodal extension (ENE) in patients who are node positive may provide prognostic information in certain human epithelial malignancies, including breast cancer $(10,11)$, squamous cell carcinoma of the vulva $(12,13)$, gastric carcinoma (14), and squamous carcinoma of the head and neck (15-21). Others refuted the significance of ENE (22-26). ENE is a frequent histologic finding in lymph nodes with prostate cancer, but its prognostic significance has not been established. We previously demonstrated that nodal cancer volume is the single most important predictor of systemic progression-free survival 
in patients who are node positive and treated by radical retropubic prostatectomy, bilateral pelvic lymphadenectomy, and androgen deprivation (8). In this study, we evaluated the impact of ENE on survival and its association with other clinical and pathologic characteristics in a large number of patients who were node positive.

\section{PATIENTS AND METHODS}

\section{Patients}

The study population consisted of the Mayo Clinic patients who underwent radical retropubic prostatectomy and bilateral pelvic lymphadenectomy between January 1987 and December 1992. All patients had regional lymph node metastasis at the time of surgery and were treated with androgen deprivation therapy within 90 days of radical prostatectomy. Exclusion criteria from the study were 1) preoperative treatment with androgen deprivation, 2) no androgen deprivation therapy within 90 days of radical prostatectomy, 3) cancer volume of primary tumors not available, 4) DNA ploidy analysis not performed, or 5) histologic slides of lymph node metastasis not available for evaluation. The final study group consisted of 212 patients.

Patients were evaluated quarterly for the first 2 years, semiannually for 2 more years, and then annually. Follow-up examinations after surgery included physical examination, serum prostate-specific antigen (PSA) measurements, chest radiography, and computerized tomography of the abdomen and pelvis, as clinically indicated $(8,9)$. Radionuclide bone scanning was performed at least annually or as clinically indicated. Serum PSA was measured using the Hybritech Tandem-R PSA assay (Hybritech, Inc., San Diego, CA) in all patients. In patients who underwent follow-up at another institution, PSA concentration was determined at the Mayo Clinic by means of a mailed blood specimen, or the patients were contacted annually and additional medical information was obtained from the local physician, if necessary.

\section{Specimens}

The radical prostatectomy and bilateral pelvic lymphadenectomy specimens were examined by frozen section at operation and subsequently by permanent sections, as previously described (8). Briefly, the apex and base of the prostate were amputated or submitted as en face (shave) margins, and the prostate was serially sectioned perpendicular to the long axis of the gland from the apex to the tip of the seminal vesicles. After gross examination of the whole prostate slices, frozen sections were selected to encompass the cancer; the length, width, and height were determined by microscopic examination of fro- zen sections. The number of cancer sections submitted for frozen examinations from the radical prostatectomy specimens varied from 8 to 20 in this series, depending on cancer volume, prostate volume, and the preference of the pathologist. Approximately 14 prostate blocks were examined per case, and the method of sampling remained constant during the study period (8). All histologic evaluations were performed without knowledge of the clinical outcome. The 1997 TNM (tumor, lymph nodes, metastasis) system was used for pathologic staging $(27,28)$. Pathologic stages were pT2a (8 patients), pT2b (14 patients), pT3a (35 patients), and pT3b (155 patients). Grading of the primary cancer was performed according to the Gleason system (29) and was based on retrospective review of all available histologic slides by authors (LC, JCC, and DGB). All tumors were examined. Tumor heterogeneity is accounted for by assigning a primary pattern of the dominant grade and a secondary pattern for the nondominant grade; Gleason score was obtained by the summation of these two histologic pattern. Gleason score was 6 (13 patients), 7 (118 patients), 8 (17 patients), 9 (54 patients), and 10 (1 patient). Prostatectomy specimens were examined for deoxyribonucleic acid (DNA) ploidy by flow cytometry with the Hedley technique. DNA ploidy analysis was performed in all patients, and DNA histograms were classified as diploid (101 patients), tetraploid (84 patients), and aneuploid (27 patients).

Bilateral pelvic lymph node dissection was performed using the modified approach, including excision of inferior chain of the external iliac lymphatics and the obturator and hypogastric nodes. The lymph nodes were totally embedded for histologic evaluation. The median number of lymph nodes sampled during bilateral open pelvic lymph node dissection was 14 (range, 4 to 33). The number of positive nodes were one (98 patients), two (55 patients), three (27 patients), four (10 patients), and five or more (22 patients). ENE was defined as cancer perforating the capsule into the perinodal tissue (Fig. 1). The presence of cancer cells within the capsule was not considered ENE. The authors recognized the difficulty in distinguishing whether metastatic cancer is within the lymph node or extends into surrounding adipose tissue because pelvic lymph nodes are extremely fatty. Metastatic deposits within fats were counted as ENE. Nodal cancer volume (size) was determined in permanent sections by the grid method (30), and the total cancer volume of all positive nodes (nodal cancer volume) was used for analysis (8). Nodal cancer volume ranged from 0.01 to $3.05 \mathrm{~cm}^{3}$ (mean, $0.23 \mathrm{~cm}^{3}$; median, $0.06 \mathrm{~cm}^{3}$ ).

\section{Statistical Analysis}

Survival was estimated using the Kaplan-Meier method. The Cox proportional hazards model was 

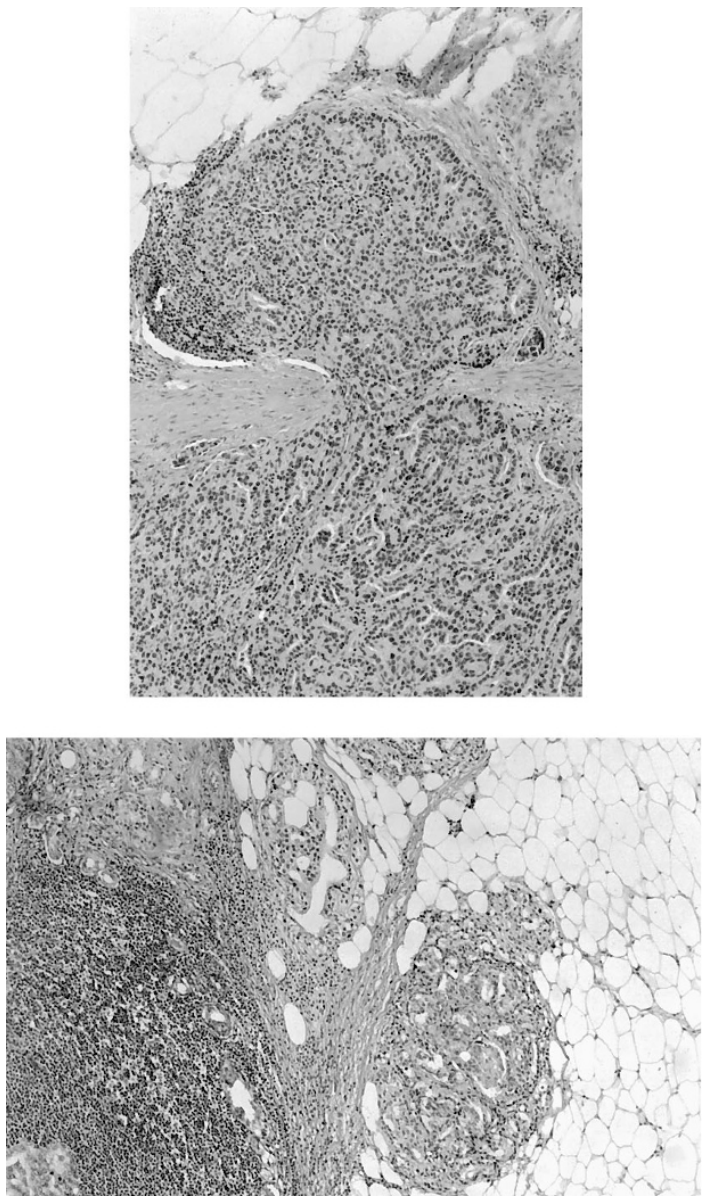

FIGURE 1. Extranodal extension in lymph node metastasis from prostate cancer.

used to test for univariate associations of ENE with survival. The influence of ENE on survival was tested after controlling for known risk factors in patients who were node positive (Gleason score, DNA ploidy, and lymph node cancer volume). Anal- ysis of association of continuous variables with survival were performed using single degree of freedom (linear) terms in the Cox model. Comparison of clinicopathologic characteristics between patients with ENE and patients without ENE was assessed using rank sum tests. A $P$ value of less than 0.05 was considered significant, and all $P$ values were two tailed.

\section{RESULTS}

Patients ranged in age from 47 to 79 years (mean, 66 years). During the mean follow-up interval of 6.3 years (median, 6.1 years; range 0.03 to 10.5 years), 23 patients developed systemic progression (distant metastasis), 18 died of prostate cancer, and 17 patients died of other causes. Five-year systemic progression-free and cancer-specific survivals were $91 \%( \pm 2 \%)$ and $95 \%( \pm 5 \%)$, respectively.

ENE was observed in 126 of 212 patients (59\%). Fifty patients $(23 \%)$ had two or more positive nodes that showed ENE. The presence of ENE was not predictive of distant metastasis-free survival or cancer-specific survival (Fig. 2). Patients with ENE involving one node had similar prognosis as those with ENE in more than one node. Five-year actuarial distant metastasis-free and cancer-specific survivals were $94 \%$ and $95 \%$, respectively, for patients without ENE, compared with $89 \%$ and $95 \%$ for those with $\operatorname{ENE}(P=.29$ and $P=.15$, respectively). For 98 patients with only one positive lymph node, there was no difference in terms of distant metastasis-free or cancer-specific survivals among patients with or without ENE (Fig. 3). Among patients with only one positive node, 5-year actuarial distant metastasis-free and cancer-specific survivals were $96 \%$ and $98 \%$, respectively, for patients
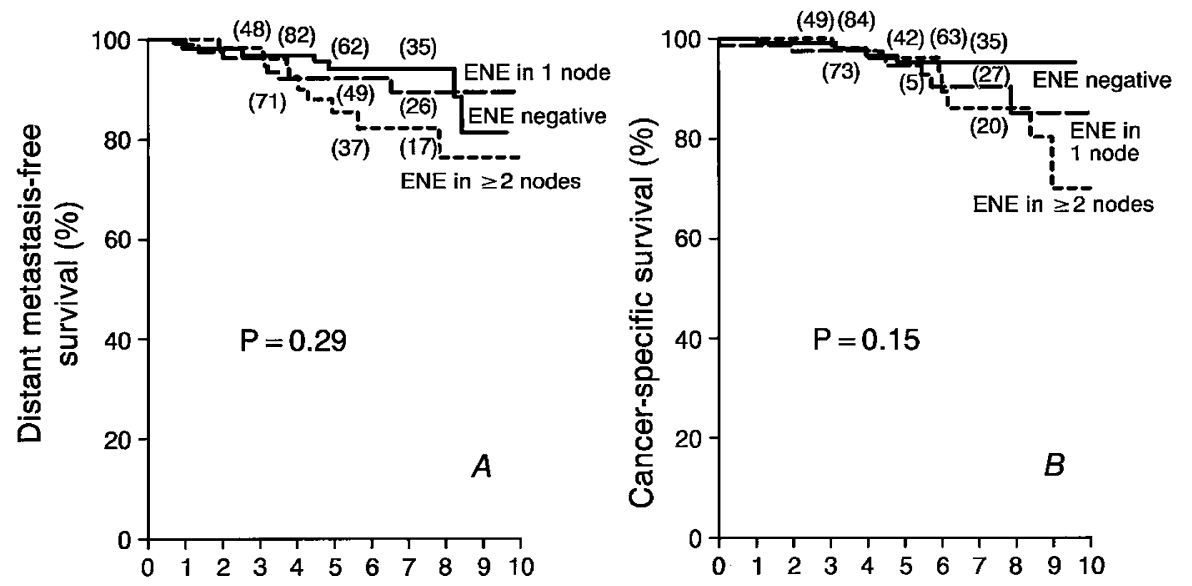

Time from prostatectomy (years)

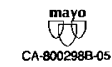

FIGURE 2. Kaplan-Meier curves (distant metastasis-free and cancer-specific survival) for 212 patients who were lymph node positive according to the number of nodes with extranodal extension. Numbers within parentheses represent number of patients still under observation at 3 , 5 , and 7 years. 


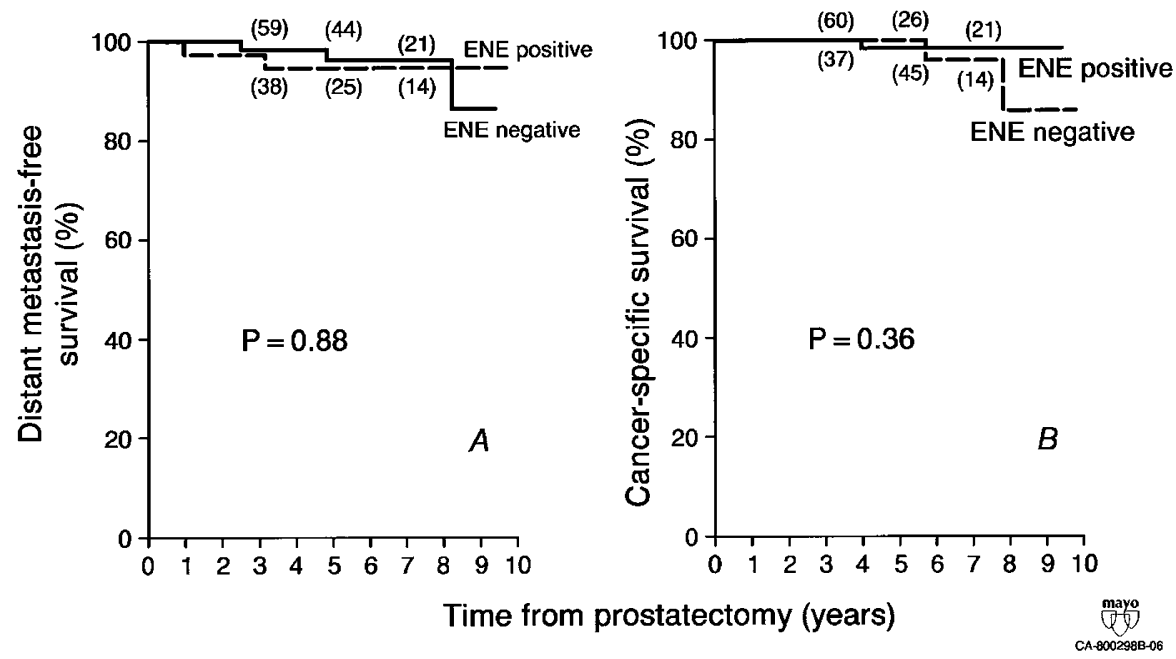

FIGURE 3. Kaplan-Meier curves (distant metastasis-free and cancer-specific survival) for 98 patients with single positive lymph node. Numbers within parentheses represent number of patients still under observation at 3, 5, and 7 years.

without ENE, compared with $95 \%$ and $100 \%$ for those with ENE ( $P=.88$ and $P=.36$, respectively).

ENE was associated with higher preoperative PSA concentration, pathologic stage, Gleason score, the number of positive nodes, the largest dimension of nodal metastasis, and nodal cancer volume (Table 1). In a multivariate analysis, ENE was not significantly associated with adverse survival, after adjusting for Gleason score, DNA ploidy, and nodal cancer volume (Table 2). Only nodal cancer volume was associated with poor survival $(P<.001$, Table 2$)$

\section{DISCUSSION}

We found that more than half of the patients who had prostate cancer with regional lymph node me-

TABLE 1. Comparison of Clinical and Pathologic Findings Between Patients with Extranodal Extension and without Extranodal Extension

\begin{tabular}{lccc}
\hline \multirow{2}{*}{ Variables } & \multicolumn{2}{c}{ Median Value } & \\
\cline { 2 - 3 } & $\begin{array}{c}\text { Negative ENE } \\
(N=126)\end{array}$ & $\begin{array}{c}\text { Positive ENE } \\
(N=86)\end{array}$ & \\
\hline Age & 67 & 66 & .66 \\
Preoperative PSA levels (ng/ml) & 17.1 & 27.1 & $<.001$ \\
Pathologic stage & & & .005 \\
$\quad$ T2 & 68 & 32 & \\
T3 & 37 & 63 & \\
Gleason score & & & .047 \\
$\quad 4-6$ & 69 & 31 & \\
7 & 42 & 58 & \\
$\quad 8-10$ & 35 & 65 & .10 \\
DNA ploidy & & & \\
$\quad$ Diploid & $52 \%$ & $43 \%$ & \\
$\quad$ Teteraploid & $40 \%$ & $42 \%$ & \\
$\quad$ Aneuploid & $8 \%$ & $15 \%$ & \\
The number of lymph nodes & 14 & 14 & .65 \\
$\quad$ sampled & & & \\
The number of positive nodes & 1 & 2 & $<.001$ \\
The largest dimension of nodal & 0.2 & 0.6 & $<.001$ \\
$\quad$ metastasis (cm) & & & \\
Nodal cancer volume (cc) & .03 & .18 & $<.001$ \\
\hline
\end{tabular}

ENE, extranodal extension; PSA, prostate-specific antigen.

${ }^{a} P$-value was obtained from the rank sum tests. tastasis had ENE. Although the presence of ENE was correlated with preoperative PSA concentration, Gleason score, pathologic stage, and nodal cancer volume, ENE was not associated with worse patient outcome in terms of distant metastasis-free or cancer-specific survival. Conversely, nodal cancer volume provided significant prognostic information, and we recommend that nodal cancer volume (or, as a surrogate, the diameter of largest metastasis) be evaluated in patients with lymph node metastasis.

In a study of 69 patients who were node positive, Griebling et al. (31) found that $55 \%$ of patients had evidence of ENE, similar to our findings (59\%). With a mean follow-up of 2.9 years, 19 patients (28\%) died of prostate cancer. They found that Gleason score and ENE were independent predictors of cancer-specific survival in patients with nodepositive prostate cancer. However, the presence of ENE was not associated with reduced survival duration in the present study. The inconsistencies with respect to the prognostic significance of ENE may be related to differences in the size of the study population, duration of follow-up, statistical methods, patient factors, primary cancer characteristics, and the possible influence of therapeutic differences. In Griebling et al.'s (31) study, only 11 patients underwent radical prostatectomy. In the present case series, all patients were treated with radical retropubic prostatectomy, bilateral pelvic lymphadenectomy, and androgen deprivation. Furthermore, only 18 patients $(8 \%)$ died of prostate cancer (mean follow-up, 6.3 years) in this study.

We previously demonstrated that nodal cancer volume could accurately predict the biologic aggressiveness of node-positive prostate cancer (8). The risk of distant metastases in patients with tumor involvement of regional lymph nodes increased proportionally with increasing nodal can- 
TABLE 2. Risk Ratios ( $\pm 95 \%$ Confidence Intervals) for Risk Factors in Node-Positive Prostate Cancer

\begin{tabular}{|c|c|c|c|c|c|c|c|c|}
\hline \multirow{3}{*}{ Characteristics } & \multicolumn{4}{|c|}{ Univariate Analysis } & \multicolumn{4}{|c|}{ Multivariate Analysis } \\
\hline & \multicolumn{2}{|c|}{ Distant metastasis } & \multicolumn{2}{|c|}{ Cancer-specific death } & \multicolumn{2}{|c|}{ Distant metastasis } & \multicolumn{2}{|c|}{ Cancer-specific death } \\
\hline & $\mathrm{RR}$ & $95 \% \mathrm{CI}$ & $\mathrm{RR}$ & $95 \% \mathrm{CI}$ & RR & $95 \% \mathrm{CI}$ & $\mathrm{RR}$ & $95 \% \mathrm{CI}$ \\
\hline Gleason grade & 1.6 & $1.1-2.4$ & 1.6 & $1.1-2.5$ & 1.1 & $0.7-1.7$ & 1.2 & $0.8-2.0$ \\
\hline DNA ploidy $^{a}$ & 1.6 & $0.7-3.8$ & 1.3 & $0.5-3.4$ & 0.9 & $0.4-2.2$ & 0.9 & $0.3-2.4$ \\
\hline Nodal cancer volume ${ }^{b}$ & 1.7 & $1.4-2.0$ & 1.4 & $1.2-1.8$ & 1.9 & $1.5-2.6$ & 1.4 & $1.1-1.9$ \\
\hline Extranodal extension & 1.6 & $0.7-3.9$ & 2.2 & $0.7-6.8$ & 0.3 & $0.1-0.9$ & 0.8 & $0.2-3.0$ \\
\hline
\end{tabular}

$\mathrm{RR}$, risk ratio; CI, confidence interval.

${ }^{a}$ Diploid $=0$, nondiploid $=1$.

${ }^{b}$ Log-2 scale, RR represents risk increase associated with a doubling in cancer volume.

cer volume. Nodal cancer volume was the best predictor of 5-year distant progression-free survival among various clinical and pathologic factors. It correlated with the established prognostic factors of the primary cancer, including Gleason score, DNA ploidy, and size of primary cancer. In that study, all patients with a nodal cancer volume of less than $0.02 \mathrm{cc}$ were free of distant disease progression (8). These findings suggest that nodal cancer volume was closely linked to the biologic behavior of metastatic prostate cancer. On the basis of these findings, we recommend that the nodal cancer volume (or, as a surrogate, the largest dimension of nodal metastases) be reported in patients with regional lymph node metastasis.

The present study may have several potential limitations. Patient follow-up was relatively short, and the sample size and the number of outcome events were limited. Some variables that were inconclusive as a result of the limited statistical power may attain statistical significance if the sample size is increased. For example, Gleason grade was significantly associated with distant metastasis-free survival (risk ratio, 1.6; 95\% confidence interval, 1.1 to 2.4) and cancer-specific survival (risk ratio, 1.6; $95 \%$ confidence interval, 1.1 to 2.5 ) in univariate analysis but was not significant after adjusting for nodal cancer volume (Table 1). The significance of Gleason grade on survival needs to be evaluated in the context of limited outcome events (18) and sample size in multivariate analysis. At the Mayo Clinic, most patients who were lymph node positive and treated with radical prostatectomy also underwent immediate adjuvant hormonal therapy. The significance of ENE may differ in patients who are treated with other approaches. Although we did not find an association between ENE and reduced survival, ENE correlated with several known predictive factors, such as preoperative PSA level, pathologic stage, Gleason score, the number of positive nodes, the largest dimension of nodal metastasis, and nodal cancer volume. Further investigation of ENE is indicated to clarify its role in cancer progression.

In summary, the presence of ENE does not seem to be associated with adverse outcome in patients who have prostate cancer with regional lymph node metastasis and who are treated by radical retropubic prostatectomy, bilateral pelvic lymphadenectomy, and androgen deprivation therapy. Conversely, nodal cancer volume (or, as a surrogate, the diameter of largest metastasis) provided useful predictive information and should be reported.

\section{REFERENCES}

1. Austenfeld MS, Davis BE. New concept in the treatment of stage D1 adenocarcinoma of the prostate. Urol Clin North Am 1990;17:867-84.

2. Ekman P. Dilemma of microscopic lymph node metastases in human prostate cancer. Eur Urol 1993;24(Suppl 2):57-60.

3. Cheng WS, Bergstralh EJ, Zincke H. Stage D1 prostate cancer: a nonrandomized comparison of conservative treatment options versus radical prostatectomy. Cancer 1993;71:9961004.

4. Zincke H, Bergstralh EJ, Larson-Keler J, Farrow GM, Myers R, Lieber MM, et al. Stage D1 prostate cancer treated by radical prostatectomy and adjuvant hormonal treatment: evidence of favorable survival in patients with diploid tumors. Cancer 1992;70:311-23.

5. Zincke $H$. The role of pathologic variables and hormonal treatment after radical prostatectomy for stage D1 disease. Oncology 1991;5:129-39.

6. Smith PH. Deferred therapy in patients with advanced disease. Cancer 1993;71:1074-7.

7. Hanks GE. The challenge of treating node-positive prostate cancer: an approach to resolving the questions. Cancer 1993; 71:1014-8.

8. Cheng L, Bergstralh EJ, Cheville JC, Slezak J, Corica FA, Zincke $\mathrm{H}$, et al. Cancer volume of lymph node metastasis predicts progression in prostate cancer. Am J Surg Pathol 1998;22:1491-500.

9. Seay TM, Blute ML, Zincke H. Long-term outcome in patients with pTxN+ adenocarcinoma of prostate treated with radical prostatectomy and early androgen ablation. J Urol 1998;159:357-64.

10. Fisher ER, Gregorio RM, Redmond C, Kim WS, Fisher B. Pathologic findings from the National Surgical Adjuvant Breast Project (Protocol No. 4): III. The significance of extranodal extension of axillary metastases. Am J Clin Pathol 1976;65:439-44.

11. Fisher BJ, Perera FE, Cooke AL, Opeitum A, Dar AR, Venkatesan VM, et al. Extracapsular axillary node extension in patients receiving adjuvant systemic therapy: an indication for radiotherapy. Int J Radiat Oncol Biol Phys 1997;38:551-9.

12. Paladini D, Cross P, Lopes A, Monaghan JM. Prognostic significance of lymph node variables in squamous cell carcinoma of the vulva. Cancer 1994;74:2491-6.

13. Origoni M, Sideri M, Garsia S, Carinelli SG, Ferrari AG. Prognostic value of pathological pattern of lymph node positivity 
in squamous cell carcinoma of the vulva stage II and IVA FIGO. Gynecol Oncol 1992;45:313-6.

14. Giorgia AD, Botti C, Sammartina P, Mingazzni P, Flammia M, Stipa V. Extracapsular lymph node metastases in the staging and prognosis of gastric cancer. Int Surg 1991;76: 218-21.

15. Snow GB, Annyas AA, Van Slooten EA, Bartelink H, Hart AA. Prognostic factors of neck node metastasis. Clin Otolaryngol 1982;7:185-92.

16. Hirabayashi H, Koshi K, Kohei U, Ohgaki H, Nakasone Y, Fujisawa T, et al. Extracapsular spread of squamous cell carcinoma in neck lymph nodes: prognostic factor of laryngeal cancer. Laryngoscope 1991;101:502-6.

17. Johnson JT, Myers EN, Bedetti CD, Barnes L, Schramm VL, Thearle PB. Cervical lymph node metastasis: incidence and implications of extracapsular carcinoma. Arch Otolaryngol 1985;111:534-7.

18. Richard JM, Sancho-Garnier H, Micheau C, Saravane D, Cachin Y. Prognostic factors in cervical lymph node metastasis in upper respiratory and digestive tract carcinoma: a study of 1713 cases during a 15-year period. Laryngoscope 1987;97:97-101.

19. Umeda M, Nishimatsu N, Teranobu O, Shimada K. Criteria for diagnosing lymph node metastasis from squamous cell carcinoma of the oral cavity: a study of the relationship between computed tomographic and histologic findings and outcome. J Oral Maxillofac Surg 1998;56:588-93.

20. Valle-Zapico AD, Fernandez FF, Suarez AR, Angul CM, Quintela JR. Prognostic value of histopathologic parameters and DNA ploidy flow cytometry in squamous cell carcinoma of the pyriform sinus. Laryngoscope 1998;108:269-72.

21. Medini E, Medini A, Lee C, Gapany M, Levitt SH. The management of metastatic squamous cell carcinoma in cervical lymph nodes form an unknown primary. Am J Clin Oncol 1998;21:121-5.

22. Donegan W, Stine SB, Samter TG. Implications of extracapsular nodal metastasis for treatment and prognosis of breast cancer. Cancer 1993;72:778-82.

23. Mambo NC, Gallager HS. Carcinoma of the breast: the prognostic significance of extranodal extension of axillary disease. Cancer 1977;39:2280-5.

24. Monsour PD, Sause WT, Avent JM, Noyes RD. Local control following therapeutic nodal dissection for melanoma. J Surg Oncol 1993;54:18-22.

25. Clayton F, Hopkins CL. Pathologic correlates of prognosis in lymph node-positive breast carcinomas. Cancer 1993;71:178290.

26. Cybulsky IJ, Lanza LA, Putnam JB, McMurtrey MM, Roth JA. Prognostic significance of computed tomography in resected N2 lung cancer. Annu Thoracic Surg 1992;54:533-7.

27. Bostwick DG. Staging prostate cancer-1997: current methods and limitations. Eur Urol 1997;32(Suppl 3):2-14.

28. Fleming ID, Coper JS, Henson DE, Hutter RVP, Kennedy BJ, Murphy GP, et al., editors. Cancer staging manual (American Joint Committee on Cancer). 5th ed. Philadelphia: RavenLippincott; 1997.

29. Bostwick DG. Neoplasms of the prostate. In: Bostwick DG, Eble JN, editors. Urologic surgical pathology. 1st ed. St. Louis: Mosby, 1997:343-422.

30. Humphrey PA, Vollmer RT. Intraglandular tumor extent and prognosis in prostatic carcinoma: application of a grid method to prostatectomy specimens. Hum Pathol 1990;21: 799-804.

31. Griebling TL, Ozkutlu D, See WA, Cohen MB. Prognostic implications of extracapsular extension of lymph node metastases in prostate cancer. Mod Pathol 1997;10:804-9.

\section{Book Review}

\section{Spector TD, Axford JS: An Introduction to Gen- eral Pathology, 4th Edition, Edinburgh, Churchill Livingstone, 391 pp, 1999 (\$39).}

This book was dedicated to the late Professor W.G. Spector, who wrote the first two editions and is remembered by the present authors as "one of the first teachers to believe that understanding rather memorizing the key disease processes is the best way to learn pathology." Written in the same spirit, this fourth edition is a worthy descendant of its predecessors, continuing the time-honored tradition of British teaching at its best.

This slim volume, published in a pocket-size format, was prepared for medical students enter- ing pathology. It covers the classic topics of general pathology, and, so far as I am concerned, it could serve as an ideal text for those 3- to 4-week-long introductory courses that were once called "Mechanism of Disease" or "Pathobiology." In the "modern" curricula dominating the U.S. medical school teaching, unfortunately, few medical students will find time to read it. I hope, however, that the book will not be unnoticed by their professors, especially those who subscribe to Professor Spector's credo.

\section{Ivan Damjanov \\ University of Kansas School of Medicine Kansas City, Kansas}

